\title{
GRP78 overproduction in pancreatic beta cells protects against high-fat-diet-induced diabetes in mice
}

\author{
T. Teodoro-Morrison • I. Schuiki • L. Zhang • D. D. Belsham • A. Volchuk
}

Received: 4 July 2012 / Accepted: 21 January 2013 / Published online: 9 March 2013

(C) Springer-Verlag Berlin Heidelberg 2013

\begin{abstract}
Aims/hypothesis Endoplasmic reticulum (ER) stress has been detected in pancreatic beta cells and in insulinsensitive tissues, such as adipose and liver, in obesitylinked rodent models of type 2 diabetes. The contribution of ER stress to pancreatic beta cell dysfunction in type 2 diabetes is unclear. We hypothesised that increased chaperone capacity protects beta cells from ER stress and dysfunction caused by obesity and improves overall glucose homeostasis.

Methods We generated a mouse model that overproduces the resident ER chaperone GRP78 (glucose-regulated protein $78 \mathrm{kDa}$ ) in pancreatic beta cells under the control of a rat insulin promoter. These mice were subjected to high-fat diet (HFD) feeding for 20 weeks and metabolic variables and markers of ER stress in islets were measured.

Results As expected, control mice on the HFD developed obesity, glucose intolerance and insulin resistance. In contrast, GRP78 transgenic mice tended to be leaner than their non-transgenic littermates and were protected against development of glucose intolerance, insulin resistance and ER stress in islets. Furthermore, islets from transgenic mice had
\end{abstract}

T. Teodoro-Morrison and I. Schuiki contributed equally to this study.

T. Teodoro-Morrison · I. Schuiki $\cdot$ L. Zhang $\cdot$ A. Volchuk $(\bowtie)$

Division of Cellular and Molecular Biology, Toronto General

Research Institute, University Health Network,

101 College Street, TMDT 10-706,

Toronto, ON, Canada M5G 1L7

e-mail: avolchuk@uhnres.utoronto.ca

T. Teodoro-Morrison $\cdot$ A. Volchuk

Department of Biochemistry, University of Toronto,

Toronto, ON, Canada

D. D. Belsham · A. Volchuk

Department of Physiology, University of Toronto,

Toronto, ON, Canada a normal insulin content and normal levels of cell-surface GLUT2 (glucose transporter 2) and the transgenic mice were less hyperinsulinaemic than control mice on the HFD. Conclusions/interpretation These data show that increased chaperone capacity in beta cells provides protection against the pathogenesis of obesity-induced type 2 diabetes by maintaining pancreatic beta cell function, which ultimately improves whole-body glucose homeostasis.

Keywords Endoplasmic reticulum stress · Obesity · Pancreatic beta cell $\cdot$ Type 2 diabetes

$\begin{array}{ll}\text { Abbreviations } \\ \text { ER } & \text { Endoplasmic reticulum } \\ \text { EIF2 } \alpha & \text { Eukaryotic initiation factor } 2 \alpha \text { subunit } \\ \text { GLUT2 } & \text { Glucose transporter 2 protein } \\ \text { GRP78 } & \text { Glucose-regulated protein } 78 \mathrm{kDa} \\ \text { GSIS } & \text { Glucose-stimulated insulin secretion } \\ \text { HFD } & \text { High-fat diet } \\ \text { IPGTT } & \text { Intraperitoneal glucose tolerance test } \\ \text { RIP } & \text { Rat insulin promoter } \\ \text { TCP } & \text { Toronto Centre for Phenogenomics } \\ \text { UPR } & \text { Unfolded protein response }\end{array}$

\section{Introduction}

Hyperglycaemia in type 2 diabetes is a consequence of insulin secretion being insufficient to maintain normal glucose homeostasis. This can result from reduced pancreatic beta cell function (glucose-stimulated insulin secretion, GSIS) and/or a reduction in pancreatic beta cell mass [1]. Obesity is the greatest risk factor for the development of the disease $[2,3]$.

The molecular mechanisms that cause pancreatic beta cell failure in the context of obesity are complex, although 
several factors have been identified. In addition to genetic predisposition [4], factors associated with obesity, including elevated NEFA and glucose, create various cellular stresses. The combination of excess NEFA and glucose (known as glucolipotoxicity [5]) can lead to increased oxidative stress, which can impair insulin biosynthesis and secretion. Furthermore, glucolipotoxicity can also induce insulin resistance, which forces the pancreatic beta cell to compensate by producing and secreting excess insulin. This causes stress in the endoplasmic reticulum (ER), which is exacerbated in an environment of excess NEFA. Finally, obesity is associated with chronic low-level inflammation, which can induce both insulin resistance and ER stress $[2,6,7]$.

ER stress has been implicated in obesity-linked type 2 diabetes since markers of the unfolded protein response (UPR), which counteract ER stress [8], have been found to be elevated in liver, adipose tissue and pancreatic islets in rodent models of obesity and diabetes and in humans with type 2 diabetes [9-14]. Importantly, chemical chaperones that reduce ER stress improve glycaemic control in the $o b / o b$ mouse [15] and recent studies suggest that these compounds may have some efficacy in improving insulin sensitivity in humans $[16,17]$. However, since ER stress can potentially contribute to both insulin resistance and pancreatic beta cell dysfunction, the exact cause of the improved glucose homeostasis in chemical-chaperone-treated rodent models of obesity remains unclear. The relative importance of ER stress in pancreatic beta cell dysfunction in obesityinduced diabetes is also unknown.

Glucose-regulated protein $78 \mathrm{kDa}$ (GRP78, also known as BiP or HSPA5) is an abundant ER chaperone that has numerous roles in the ER [18], among which is an essential function in the folding of newly synthesised proteins, including proinsulin in pancreatic beta cells $[19,20]$. In addition, GRP78 is an important regulator of the UPR [21]. GRP78 overproduction has been shown to reduce ER stress in cultured cell lines and in tissues in vivo $[19,22-24]$ and also to protect pancreatic beta cells from lipotoxicity in vitro $[10,25]$. To test the hypothesis that ER stress contributes to beta cell dysfunction in obesity-induced diabetes we examined glucose homeostasis in response to high-fat diet (HFD) in transgenic mice overproducing GRP78 in pancreatic beta cells.

\section{Methods}

Generation of RIP-GRP78 transgenic mice pINS-BS plasmid containing the rat insulin promoter (RIP) was provided by P. Ohashi (Ontario Cancer Institute) [26]. Human GRP78 tagged with a Myc epitope was amplified by PCR from the pCMV-GRP78-Myc-KDEL plasmid (obtained from R. Prywes, Columbia University) and subcloned into pCRII TOPO vector (Life Technologies, Burlington, ON,
Canada). The GRP78-Myc-KDEL cDNA was cut from pCR II TOPO with $\mathrm{XbaI} / \mathrm{BamHI}$ and subcloned into pINSBS. This construct was cut with KpnI/HindIII and the RIPGRP78-Myc-KDEL-polyA fragment $(\sim 4.3 \mathrm{~kb})$ was gelpurified (Gel Extraction Kit; Qiagen, Toronto, ON, Canada), followed by purification with a PCR purification kit (Qiagen). The DNA was eluted in filtered microinjection buffer and injected into zygotes from a cross between $\mathrm{C} 57 \mathrm{BL} / 6$ female and B6D2F1 $(\mathrm{C} 57 \mathrm{BL} / 6 \times \mathrm{DBA} / 2)$ male mice at the Toronto Centre for Phenogenomics (TCP). Thus, founder mice from the initial pronuclear injection contained a 75\% C57BL/6 background. Of the 33 founder mice born, 10 were positive for the GRP78-Myc-KDEL transgene. For genotyping, mouse tail DNA was isolated (DNeasy Blood \& Tissue Kit; Qiagen) and analysed by PCR (primers and PCR protocol available upon request). These mice were bred with C57BL/6 mice and the progeny were assayed for islet transgene protein production. Two strains produced GRP78-Myc in essentially all beta cells in the islets (No. 3064 and No. 3068). Before HFD implementation, these strains were bred for five or six generations with C57BL/6 mice.

HFD feeding All animal procedures were approved and performed in accordance with the Animal Use Protocols at the TCP. Mice were placed on an HFD $(45 \% \mathrm{~kJ}$ from fat; D12451 Research Diets, New Brunswick, NJ, USA) at 8 weeks of age for 20 weeks. Food consumption was determined by weighing HFD chow per cage over a $24 \mathrm{~h}$ period, divided by the number of mice per cage of the same genotype. Mice were scanned for fat content using an EchoMRI Whole Body Composition Analyzer (Echo Medical Systems, Houston, TX, USA) in the fed state at the endpoint of HFD feeding. Body fat mass was determined using EchoMRI 2008.01.18M software (Echo Medical Systems).

Tissue collection and islet isolation Mice were injected with a ketamine/xylazine mixture (100 mg per $\mathrm{kg} / 10 \mathrm{mg}$ per $\mathrm{kg}$, respectively). The pancreas was immediately removed and stored in Hanks' balanced salt solution (Life Technologies) on ice. Liver, brain and hypothalamic tissues were collected and placed immediately on dry ice and stored at $-80^{\circ} \mathrm{C}$. Islets were isolated as described previously [27]. Islet pellets ( 20 islets) were lysed directly in $20 \mu \mathrm{l} 2 \times$ Laemmli sample buffer $(+10 \%$ beta-mercaptoethanol). In some cases, islets were lysed in RIPA buffer $(150 \mathrm{mmol} / \mathrm{l} \mathrm{NaCl}, 1 \% \mathrm{NP}-40,0.5 \%$ deoxycholate, $0.1 \%$ sodium dodecyl sulphate, $50 \mathrm{mM}$ Tris, $\mathrm{pH} 8.0$ ) supplemented with protease and phosphatase inhibitors ( $0.5 \mathrm{mmol} / \mathrm{l}$ phenylmethylsulfonyl fluoride, $10 \mu \mathrm{g} / \mathrm{ml}$ aprotinin, $10 \mu \mathrm{g} / \mathrm{ml}$ leupeptin, $10 \mathrm{nmol} / \mathrm{l}$ okadaic acid, $2 \mathrm{mmol} / \mathrm{l}$ sodium vanadate, $10 \mathrm{mmol} / \mathrm{l}$ sodium fluoride) for $1 \mathrm{~h}$ on ice, followed by $10 \mathrm{~min}$ centrifugation $\left(16,100 \mathrm{~g}, 4^{\circ} \mathrm{C}\right)$. For analysis of insulin content, islets were lysed in RIPA buffer supplemented with protease inhibitors for $2 \mathrm{~h}$ on ice. A Pierce BCA protein 
assay (Thermo Scientific, Nepean, ON, Canada) was used for protein quantification. Insulin was measured using an RIA kit (Millipore, Billerica, MA, USA).

Insulin secretion assay Islets were isolated and allowed to recover for $48 \mathrm{~h}$ in RPMI 1640 media $(11.1 \mathrm{mmol} / 1$ glucose, $1 \mathrm{mmol} / \mathrm{l}$ sodium pyruvate, $10 \mathrm{mmol} / \mathrm{l}$ HEPES) supplemented with $10 \% \mathrm{FBS}, 2 \mathrm{mmol} / \mathrm{l}$-glutamine and antibiotics (penicillin-streptomycin). Islets were incubated in BMHH buffer $\left(125 \mathrm{mmol} / 1 \mathrm{NaCl}, 5.7 \mathrm{mmol} / \mathrm{K} \mathrm{KCl}, 2.5 \mathrm{mmol} / 1 \mathrm{CaCl}_{2}\right.$, $1.2 \mathrm{mmol} / 1 \mathrm{MgCl}_{2}, 10 \mathrm{mmol} / \mathrm{l} \mathrm{HEPES}, \mathrm{pH} 7.4,0.1 \% \mathrm{BSA}$, $2.8 \mathrm{mmol} / \mathrm{l}$ glucose) for $30 \mathrm{~min}$. Fifteen islets were hand-picked and placed in Eppendorf tubes in the same media for $40 \mathrm{~min}$ at $37^{\circ} \mathrm{C}$. The supernatant fraction was collected and the same islets were stimulated with BMHH buffer $(+16.7 \mathrm{mmol} / \mathrm{l}$ glucose) for $40 \mathrm{~min}$ at $37^{\circ} \mathrm{C}$. The supernatant fraction was collected and the pellets were stored at $-80^{\circ} \mathrm{C}$ until analysis. Islet DNA was isolated as described in [28] and used to normalise the secretion values. Insulin was measured by RIA. Proinsulin was measured using the Proinsulin Rat/Mouse ELISA kit (Catalogue No. 10-1232-01; Mercodia, Uppsala, Sweden).

Islet apoptosis assay Islets were isolated from mice and allowed to recover for $24 \mathrm{~h}$ in RPMI 1640 media supplemented as described above. Thirty islets per condition were treated with or without $1 \mu \mathrm{mol} / \mathrm{l}$ thapsigargin or $0.5 \mathrm{mmol} / \mathrm{l}$ palmitate $/ 0.5 \%$ BSA $(+25 \mathrm{mmol} / 1$ glucose $)$ for $48 \mathrm{~h}$. Following the treatment, islets were lysed and cytoplasmic oligonucleosomes were measured using the Cell Death Detection ELISA Kit (Catalogue No. 11774425 001; Roche, Laval, PQ, Canada). The values were normalised to islets exposed to control RPMI media. A parallel experiment was performed to determine the amount of protein in each condition using a BCA protein assay.

Pancreas fixation, immunohistochemistry and islet area analysis Pancreas tissue was fixed in 4\% paraformaldehyde/PBS at room temperature for 18-24 h. The fixed pancreas was washed with PBS and stored in $70 \%$ ethanol before being embedded in paraffin and sectioned. Pancreas sections were stained for insulin. Immunoreactivity was visualised with a horseradish peroxidase-conjugated secondary antibody and the substrate 3,3'-diaminobenzidine. Insulin-stained slides were scanned and islet area was determined by Aperio ImageScope v11.0.2.725 software (Aperio, Vista, CA, USA). For immunofluorescence microscopy, pancreas sections were deparaffinised and rehydrated with a series of xylene and ethanol washes. Slides were then permeabilised with $0.1 \%$ Triton X-100/PBS. For antigen retrieval, slides were transferred into boiled sodium citrate buffer and microwaved to boil for $30 \mathrm{~min}$, with $1 \mathrm{~min}$ intervals. Slides were incubated in blocking solution (10\% goat serum with $0.2 \%$ saponin/PBS) for $3 \mathrm{~h}$ at room temperature. Primary antibodies were: GRP78 (catalogue No. 610978; BD
Biosciences, Mississauga, ON, Canada;); Myc tag clone 9E10 (catalogue No. 05-419, Millipore); GLUT2 (catalogue No. 071402; Millipore); insulin (catalogue No. A0564; Dako, Burlington, ON, Canada). Antibodies were added in blocking solution overnight at $4^{\circ} \mathrm{C}$. Secondary antibodies, Oregon Green goat anti-mouse IgG (Life Technologies) and Cy5-conjugated donkey anti-guinea pig IgG (Jackson ImmunoResearch, West Grove, PA, USA) in blocking solution were added for $2 \mathrm{~h}$ at room temperature. Images were acquired using a Zeiss LSM510 META laser scanning confocal microscope (Zeiss, Munich, Germany) or an Olympus (IX71) fluorescence microscope (Olympus, Richmond Hill, ON, Canada) using QCapture Pro software (QImaging, Surrey, BC, Canada).

Western blotting Western blotting was performed as described previously [19]. Antibodies used: GRP78 and Myc tag (details provided above); phospho-EIF2 $\alpha$ (catalogue no. 9721; Cell Signaling Tech, Danvers, MA, USA) and $\gamma$-tubulin (catalogue No. T6557; Sigma, Aldrich, Oakville, ON, Canada). Band density from western blots was quantified using Image J 1.45 software (http://rsbweb.nih.gov/ij/index.html) and values were normalised to the $\gamma$-tubulin loading control.

RNA isolation and real-time PCR Total RNA was isolated as described previously [19]. Islet RNA (300 ng) was reverse transcribed using the High Capacity cDNA reverse transcription kit (Life Technologies, Applied Biosystems). Real-time PCR analysis was conducted as described previously [19]. Primers used (Life Technologies, Applied Biosystems) were: Grp78 (also known as Hspa5) (Mm00517690_g1), Chop (also known as Ddit3) (Mm00492097_m1), Sdf2l1 (Mm00452079_m1). The detection of spliced and unspliced mouse $X b p 1$ was performed as described previously [29].

Intraperitoneal glucose tolerance test For the intraperitoneal glucose tolerance test (IPGTT), mice were fasted overnight and the following day a $1.5 \mathrm{mg} /(\mathrm{g}$ body weight) dose of D-glucose was administered by intraperitoneal injection. Blood glucose levels were measured using a Bayer Contour Glucometer (Bayer Healthcare, Toronto, ON, Canada) at various times from tail bleeds. Plasma insulin was measured using a mouse Ultrasensitive Insulin ELISA kit (ALPCO, Salem, NH, USA).

Insulin tolerance test Mice were fasted for $5 \mathrm{~h}$ before experimentation. The mice were administered a $1 \mathrm{U} /(\mathrm{kg}$ body weight) dose of insulin (Humalog; Lilly, Indianapolis, IN, USA) by intraperitoneal injection [30] and blood glucose was measured over the next $3 \mathrm{~h}$ using blood from tail bleeds.

Statistical analysis Data are expressed as mean \pm SEM. A Student's $t$ test (two-tailed distribution, two sample assuming equal variance) was used for comparison between two groups, where $p<0.05$ denoted significance. For comparison 
among several groups a one-way ANOVA, followed by a Bonferroni or Tukey post hoc test was performed.

\section{Results}

RIP-GRP $78^{-/+}$mice have a similar metabolic phenotype to control mice Since ER stress has been implicated in pancreatic beta cell dysfunction observed in type 2 diabetes [6] we hypothesised that mice overproducing GRP78 in beta cells would be protected from developing type 2 diabetes. Therefore, we generated mice expressing Myc-tagged human GRP78 under the control of the RIP (Fig. 1a and b). Of the founder mice born, two produced progeny that expressed the transgene in essentially all pancreatic beta cells within islets. Western blot analysis revealed that the total GRP78 protein in islets from progeny of founder mouse No. 3068 (strain 3068) was approximately eightfold higher in RIP-GRP78 ${ }^{-/+}$mice a

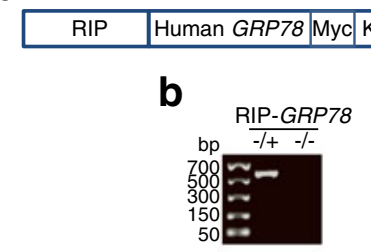

d

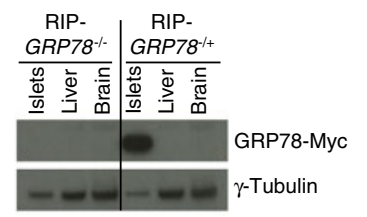

e

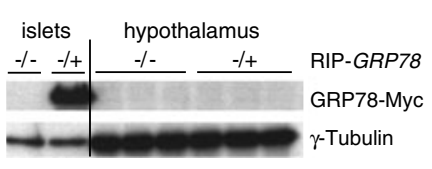

g
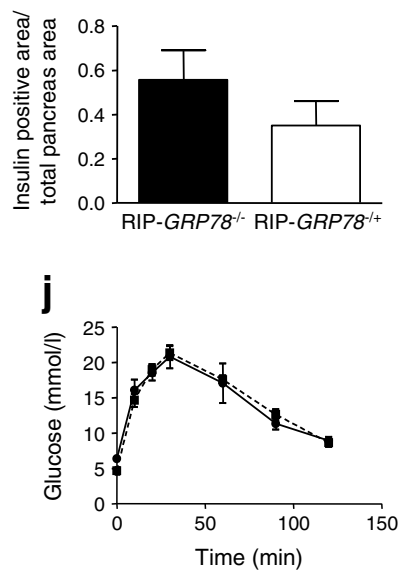

f

h
C
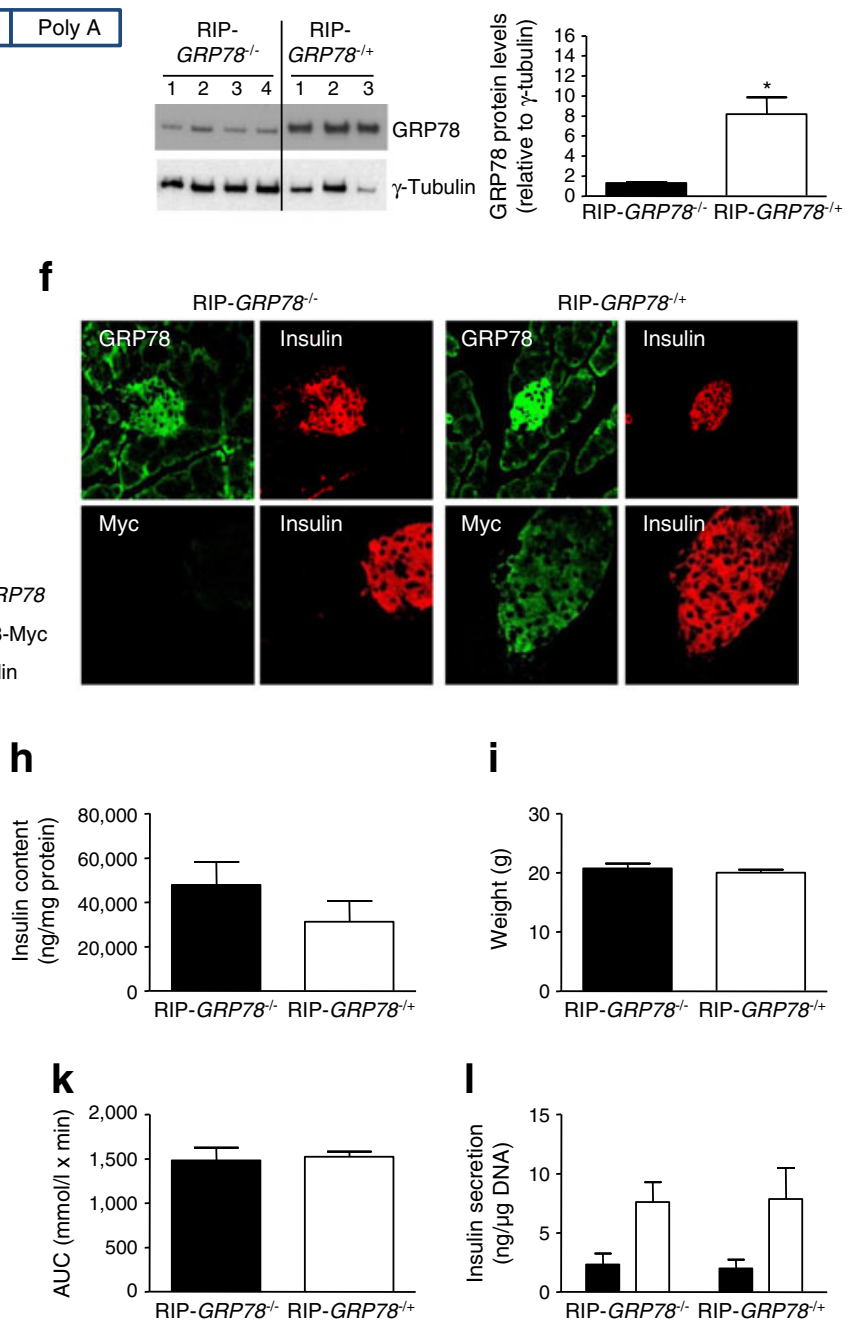

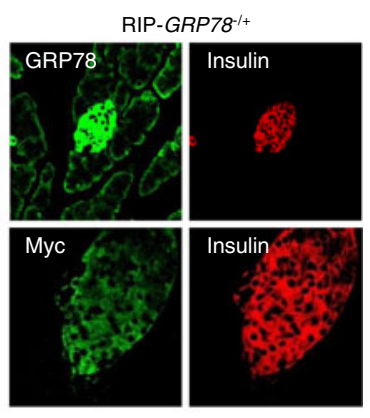

i

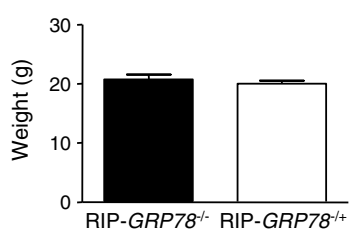

I

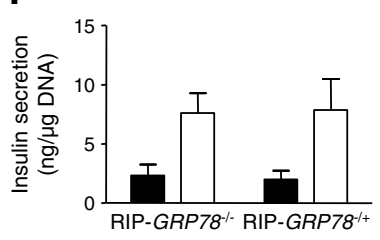

Fig. 1 Generation of RIP-GRP78 $8^{-/+}$transgenic mice and metabolic phenotype compared with transgene-negative littermates (strain No. 3068). a Schematic representation of the construct used to generate RIP-GRP78 transgenic mice. The human GRP78 protein contains a Cterminal Myc tag followed by a KDEL ER retention signal. b PCRamplified RIP-GRP78 transgene from mouse genomic DNA. c GRP78 protein levels in islets isolated from RIP-GRP7 $8^{-/-}$and RIP-GRP78 ${ }^{-/+}$ mice and quantification of relative GRP78 levels $(n=7-10$ mice per genotype, ${ }^{*} p<0.05, t$ test). d, e GRP78 transgene protein detected by an anti-Myc antibody in various tissues isolated from male RIP$G R P 78^{-/-}$and RIP-GRP $78^{-/+}$mice (liver, brain $[n=2$ mice]; hypothalamus [ $n=3$ mice of each genotype $]$ ). f Fixed pancreas sections stained with anti-Myc or anti-GRP78 and co-stained for insulin. Representative confocal images from $n=3$ mice of each genotype. $\mathbf{g}$ Insulin area per total pancreas area from 16-week-old mice $(n=3$ mice per genotype). h Insulin content in islets from 16-week-old mice $(n=3-5)$. i Mouse weight at 16 weeks of age $(n=6-9)$. j IPGTT; black circles,

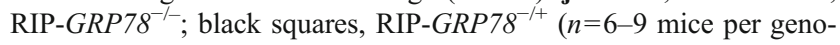
type). $\mathbf{k}$ Area under the curve (AUC) following IPGTT. I Insulin secretion in islets isolated from 16 -week-old mice $(n=4$ or 5 mice per genotype). Black bars, $2.8 \mathrm{mmol} / 1$ glucose; white bars, $16.7 \mathrm{mmol} / \mathrm{l}$ glucose. Male mice were used in $\mathbf{g}-\mathbf{k}$ 
than in their non-transgenic littermates (Fig. 1c). Using an antiMyc antibody, GRP78-Myc protein was detected in islet lysates, but not in liver, brain or hypothalamus tissues (Fig. 1d and e). Pancreas sections immunostained with a GRP78-specific antibody showed an increase in GRP78 fluorescence in beta cells (identified by insulin staining) in RIP$G R P 78^{-/+}$mice, while GRP78 staining remained similar in surrounding acinar tissue (Fig. 1f). Production of the GRP78Myc protein in islets was confirmed by immunostaining using an anti-Myc antibody (Fig. 1f). By transmission electron microscopy the beta cells from transgenic and non-transgenic mice appeared similar (not shown).

We also examined insulin-positive area in pancreas sections (reflective of beta cell mass) and insulin content in isolated islets. Neither of these variables was significantly altered in RIP-GRP $78^{-/+}$mice, compared with non-

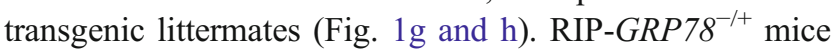
had similar weight gain to their non-transgenic littermates on a regular chow diet (Fig. 1i). Furthermore, RIP-
$G R P 78^{-/-}$and RIP-GRP78 ${ }^{-/+}$mice had identical glucose tolerance (Fig. 1j and $\mathrm{k}$ ) and GSIS from isolated islets (Fig. 11).

RIP-GRP $78^{-/+}$mice are protected from HFD-induced glucose intolerance and insulin resistance We examined whether RIP-GRP7 ${ }^{-/+}$transgenic mice are protected from diabetes resulting from HFD-induced obesity. Control and transgenic male mice were fed an HFD for 20 weeks and at the endpoint metabolic variables were examined. Compared with a standard chow diet, the HFD resulted in weight gain and increased body fat in male mice (Fig. 2a and b). Interestingly, there was a clear tendency for the RIP$G R P 78^{-/+}$mice to gain less weight (Fig. 2a) and body fat content was slightly, but significantly, reduced relative to non-transgenic mice (Fig. 2b). This difference was not due to a decrease in food consumption (Fig. 2c).

We examined glucose homeostasis in male RIP$G R P 78^{-/+}$mice and their non-transgenic littermates.
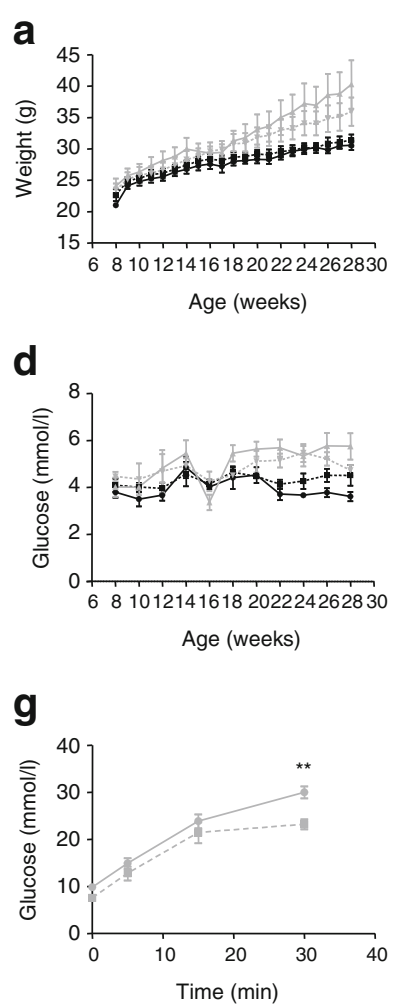

b
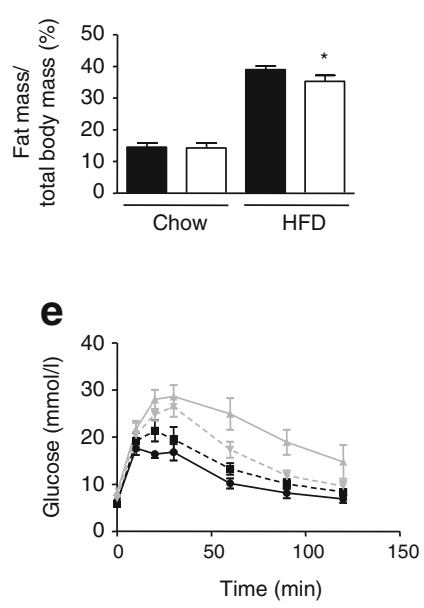

h

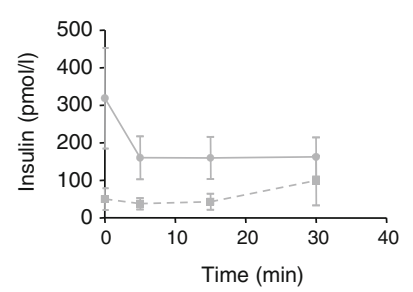

C

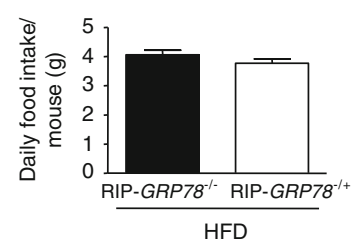

f

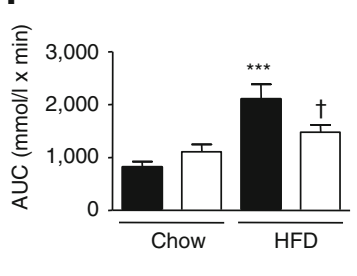

i

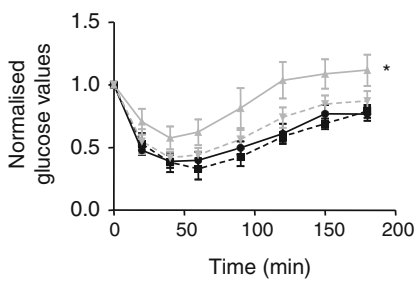

Fig. 2 Male RIP-GRP78 ${ }^{-/+}$mice (strain No. 3068) are protected against glucose intolerance and insulin resistance induced by an HFD. a Mice were placed on a control chow diet or an HFD for 20 weeks and weight was measured weekly. Black circles, RIP$G R P 78^{-/}$chow; black squares, RIP-GRP $78^{-/+}$chow; grey triangles, RIP-GRP $78^{-/-}$HFD; inverted triangles, RIP-GRP $78^{-/+}$HFD. b Mouse fat content at the endpoint of the 20 -week diet. Black bars, RIP$G R P 78^{-/-}$; white bars, RIP-GRP $78^{-/+}\left({ }^{*} p<0.05\right.$, RIP-GRP $78^{-/-}$HFD vs RIP-GRP7 $8^{-/+}$HFD, $t$ test). c Daily food consumption of HFD chow. d Bi-weekly fasting blood glucose and (e) IPGTT. Black circles, RIP-GRP $78^{-/-}$chow; black squares, RIP-GRP78 $8^{-/+}$chow; grey triangle, RIP-GRP $78^{-/-}$HFD; inverted triangles, RIP-GRP $78^{-/+}$HFD.

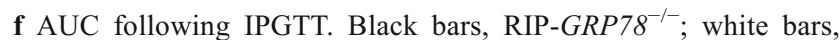
RIP-GRP $78^{-/+}\left(* * * p<0.001\right.$, RIP-GRP $78^{-/-}$chow vs RIP-GRP78 $8^{-/-}$ HFD; $\uparrow p<0.05$, RIP-GRP $78^{-/-}$HFD vs RIP-GRP $78^{-/+}$HFD, one-way ANOVA and Bonferroni post hoc test). $\mathbf{g}$ IPGTT in HFD mice and (h)

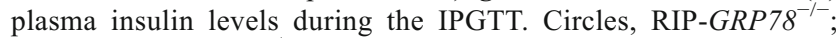
squares, RIP-GRP7 $78^{-/+}(* * p<0.05, t$ test $)$ i Insulin tolerance test. The glucose values were normalised to the starting blood glucose value for each group. Black circles, RIP-GRP78 ${ }^{-/}$chow; black squares, RIP-GRP $78^{-/+}$chow; grey triangles, RIP-GRP7 ${ }^{-/-}$HFD; inverted triangles, RIP-GRP7 $8^{-/+}$HFD (AUC, ${ }^{*} p<0.05$, RIP-GRP7 ${ }^{-/-}$chow vs RIP-GRP7 $8^{-/-}$HFD, $t$ test). Panels a, c, d-h, $n=6$ mice per condition. Panels b, i, $n=4$ mice per condition 
Fasting blood glucose tended to be lower in the transgenepositive mice compared with controls after 20 weeks on the HFD (Fig. 2d). Non-transgenic mice on the HFD were glucose intolerant compared with chow-fed mice during an IPGTT (Fig. 2e and f). RIP-GRP78 ${ }^{-/+}$mice on the HFD, however, had significantly improved glucose tolerance compared with non-transgenic littermates (Fig. 2e and f). Plasma insulin was also measured during the IPGTT in HFD mice. In contrast to control mice, transgenic mice had lower basal insulin levels and increased insulin secretion in response to glucose injection coincident with reduced serum glucose levels (Fig. $2 \mathrm{~g}$ and $\mathrm{h}$ ). We also examined insulin tolerance in the transgenic and control mice. Surprisingly, RIP$G R P 78^{-/+}$mice on the HFD had significantly better insulin tolerance than their non-transgenic littermates (Fig. 2i). In fact, insulin sensitivity was restored nearly to that observed on a regular chow diet, indicating that RIP-GRP $78^{-/+}$mice remain sensitive to insulin, despite the HFD.

In transgenic mice derived from strain No. 3064, total GRP78 protein levels in islets were approximately twofold higher compared with the levels in transgene-negative mice (Fig. 3a). Male RIP-GRP $78^{-/+}$mice derived from strain No. 3064 had similar weight at 16 weeks and glucose tolerance compared with non-transgenic littermates on a chow diet (not shown). However, male RIP-GRP $78^{-/+}$mice had reduced weight gain (Fig. 3b) and tended to have improved glucose tolerance on the HFD diet, although the change in these mice did not reach statistical significance (Fig. 3e and f). Mice from strain No. 3064 were not protected from HFDinduced insulin resistance (Fig. 3g).

Overall, these data indicate that male mice with overproduction of GRP78 in pancreatic beta cells are resistant to glucose intolerance caused by the HFD and the effect is dependent on the level of GRP78 overproduction.

RIP-GRP $78^{-/+}$mice on an HFD have more normal islets and are less hyperinsulinaemic Since RIP-GRP7 ${ }^{-/+}$mice on the HFD tended to be leaner than control mice and were protected from glucose intolerance and insulin resistance, we examined blood and islet insulin levels and islet mass. Transgene-negative mice on the HFD were characteristically hyperinsulinaemic, while RIP-GRP7 $8^{-/+}$littermates from strain No. 3068 were protected from obesity-induced hyperinsulinaemia (Fig. 4a). Furthermore, RIP-GRP7 $8^{-/+}$mice on the HFD had a normal insulin content (Fig. 4b) and a normal insulin-positive area (Fig. 4c) compared with islets from

a
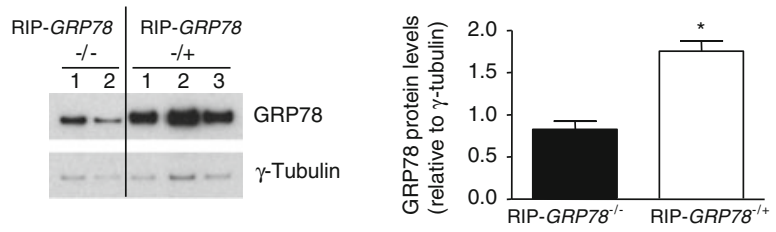

b

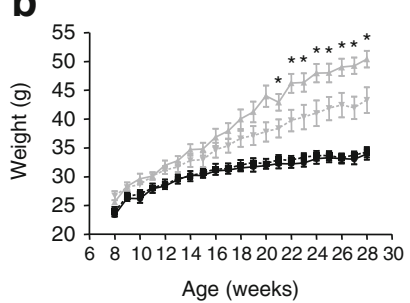

C

e

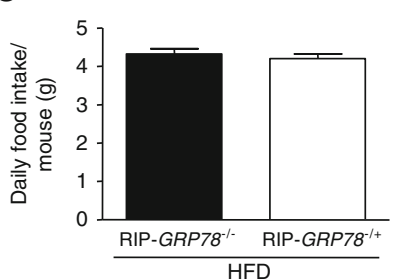

d

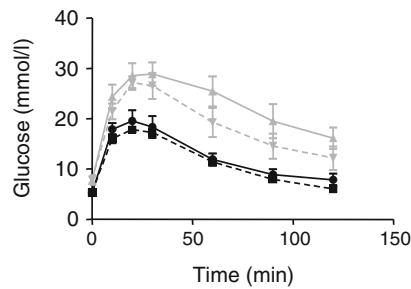

f

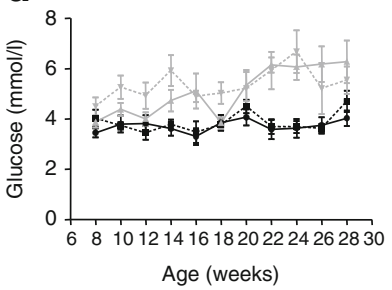

g
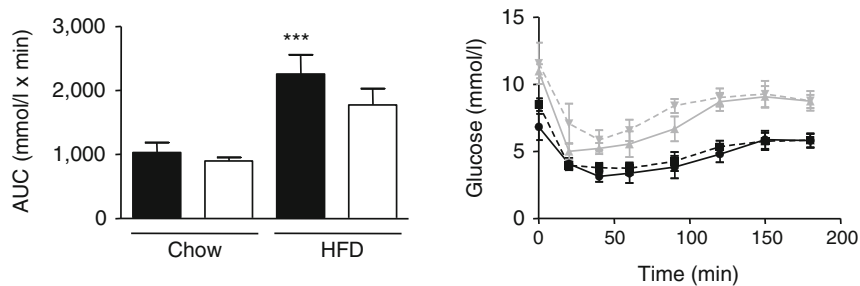

Fig. 3 Phenotype of RIP-GRP $78^{-/+}$transgenic mice derived from strain No. 3064. a GRP78 levels in islets isolated from male and female RIP-GRP $78^{-/+}$mice and transgene-negative littermates $(n=5-10$ mice per genotype, $* p<0.05 t$ test). b Male mice were fed chow or were placed on an HFD for 20 weeks and weight was measured weekly $(n=$ 6 mice for each condition; $*^{*}<0.05$, RIP-GRP7 $8^{-/-}$HFD vs RIP$G R P 78^{-/+}$HFD). c Daily food consumption of HFD per mouse.

d Bi-weekly fasting blood glucose. e IPGTT and (f) AUC following

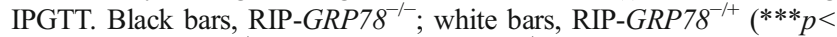

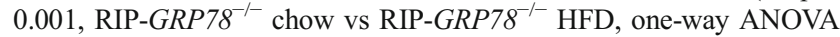
and Bonferroni post hoc test; $n=6$ mice per genotype). $\mathbf{g}$ Insulin tolerance test ( $n=3-5$ mice per genotype). In $\mathbf{b}, \mathbf{d}, \mathbf{e}, \mathbf{g}$ : black circles, RIP-GRP78 $8^{-1-}$

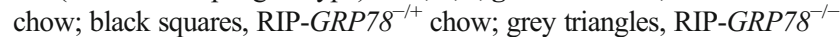
HFD; inverted triangles, RIP-GRP $78^{-/+}$HFD 
a

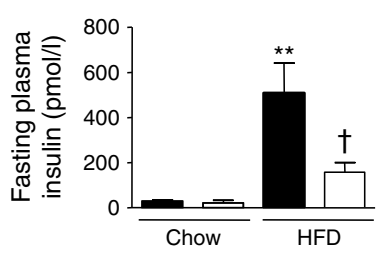

d

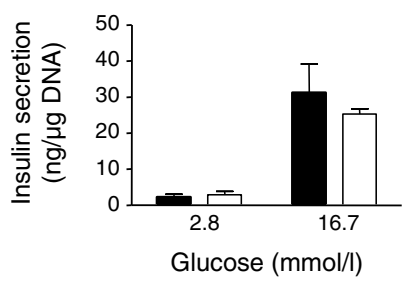

g

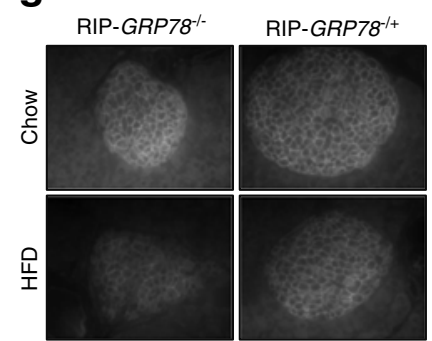

b

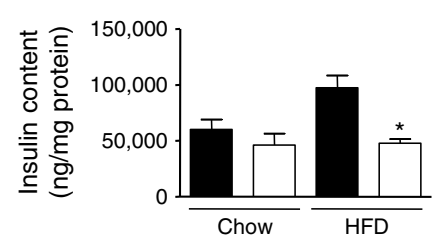

e

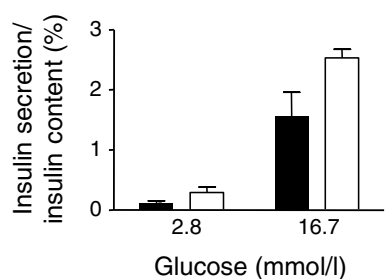

h

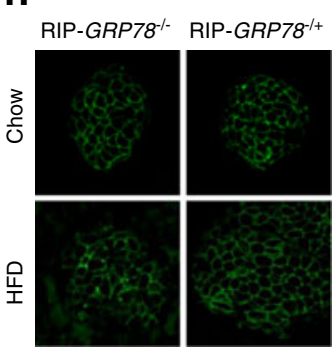

C

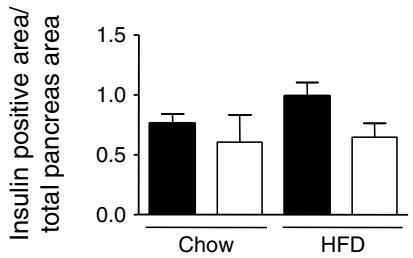

f

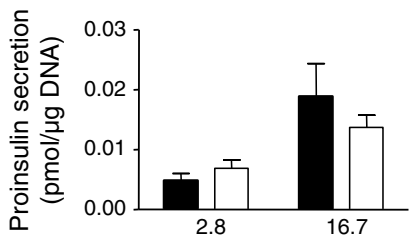

Glucose $(\mathrm{mmol} / \mathrm{l})$
Fig. 4 Transgenic RIP-GRP $78^{-/+}$mice have lower plasma insulin levels and have more normal islets on an HFD compared with transgene-negative mice. Fasting plasma insulin in male mice from strain No. 3068 (a) $\left(n=3\right.$ or 4 mice per condition, ${ }^{*} p<0.01$, RIP- $G R P 78^{-/-}$ chow vs RIP-GRP $78^{-/-}$HFD; $\uparrow p<0.05$, RIP-GRP7 $8^{-/-}$HFD vs RIP$G R P 78^{-/+}$HFD, one-way ANOVA and Tukey post hoc test), islet insulin content (b) $\left(n=4\right.$ or $5, * p<0.05$, RIP-GRP $78^{-/-}$HFD vs RIP$G R P 78^{-/+}$HFD, one-way ANOVA and Tukey post hoc test) and insulin area relative to total pancreas area (c) $(n=3-5)$. Black bars, RIP-GRP $78^{-/-}$; white bars, RIP-GRP $78^{-/+}$. d-f Islets were isolated

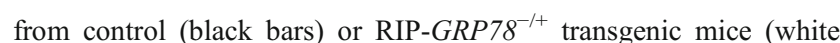
bars) following HFD feeding and GSIS was measured ( $n=3$ mice per genotype); insulin secretion relative to islet DNA (d), insulin secretion relative to islet insulin content (e) and proinsulin secretion relative to islet DNA (f). Representative images of islet GLUT2 immunofluorescence acquired using an epifluorescence microscope (g) or a confocal microscope (h) from $n=3$ mice per group. i Relative fluorescence intensity of $6-10$ islets per condition measured using NIH Image $\mathrm{J}$ software. Black bars, RIP-GRP $78^{-/-}$; white bars RIP-GRP $78^{-/+}(n=3$ mice per condition, ${ }^{*} p<0.05, t$ test) control mice, which had a significantly higher insulin content and a trend towards increased insulin-positive area. Despite having less total insulin (indeed more normal insulin levels) transgenic islets from HFD mice secreted as much insulin as control islets at stimulatory glucose concentrations (Fig. 4d). When expressed relative to islet insulin content, islets isolated from transgenic mice on the HFD had a clear trend towards better GSIS (Fig. 4e). Transgenic islets in these experiments also had a trend towards lower proinsulin secretion compared with islets from control mice (Fig. 4f), suggesting that transgenic islets may have enhanced proinsulin processing. Finally, cell-surface islet GLUT2 protein levels were reduced by chronic HFD in control mice, but the levels were maintained in islets from RIP$G R P 78^{-/+}$mice (Fig. 4g-i). Combined, these data indicate that pancreatic beta cells in RIP-GRP $78^{-/+}$transgenic mice on an HFD maintain a more normal structure and better function than beta cells from control mice on the HFD.
HFD induces mild ER stress in islets and RIP-GRP78 $8^{-/+}$ islets are protected from ER stress To determine if GRP78 overproduction protects pancreatic beta cells from HFDinduced ER stress we measured ER stress markers in isolated islets from RIP-GRP7 $78^{-/+}$mice and their transgene-negative littermates after 20 weeks on the HFD. Levels of spliced Xbpl mRNA were not changed in transgene-negative chow-fed vs HFD-fed mice (Fig. 5a and b), a result consistent with previous findings [13]. Interestingly however, islets from RIP$G R P 78^{-/+}$mice on the HFD had lower levels of spliced $X b p 1$, perhaps indicative of some protection from ER stress.

We also measured other markers of ER stress, including several known UPR target genes and proteins. Grp78 mRNA and GRP78 protein were increased in islets from control mice on HFD vs chow diet (Fig. 5c, f and g). Increased GRP78 abundance shows that the HFD resulted in enhanced chaperone levels indicative of ER stress in islets. The ER-stress-inducible gene $S d f 2 l 1$ [31, 32] was also 
a \begin{tabular}{l|l|l|l} 
RIP-GRP78 & RIP-GRP78 & RIP-GRP78 & RIP-GRP78
\end{tabular}

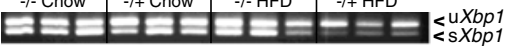

b

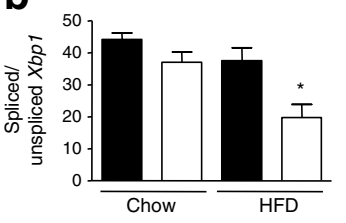

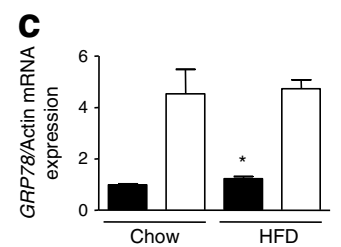

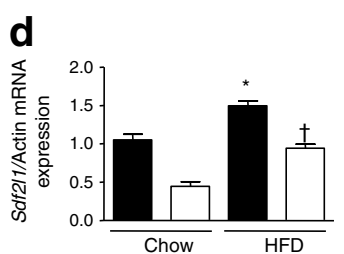

e
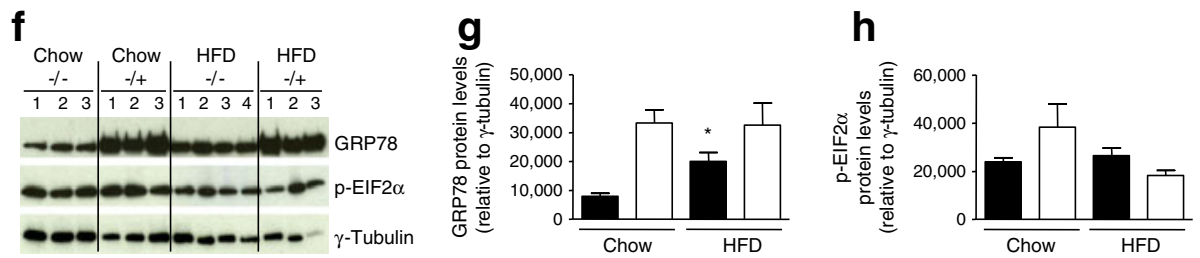

Fig. 5 HFD induces mild ER stress in islets. a Following chow or HFD feeding mouse islets were isolated and analysis of unspliced and spliced Xbpl was monitored by RT-PCR ( $n=3$ mice per group). b

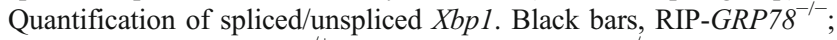
white bars, RIP-GRP7 $8^{-/+}\left({ }^{*} p<0.05\right.$, RIP-GRP $78^{-/-}$HFD vs RIP$G R P 78^{-/+}$HFD, one-way ANOVA and Tukey post hoc test). $\mathbf{c}-\mathbf{e}$ Total islet RNA analysed for ER stress markers by real-time PCR. Black bars, RIP-GRP7 $8^{-/-}$; white bars, RIP-GRP $78^{-/+}$. c Grp 78 ; note that the primers used amplify both the transgene (human GRP78) and endogenous mouse Grp78 in the RIP-GRP78 ${ }^{-/+}$samples $\left({ }^{*} p<0.05\right.$, RIP-
$G R P 78^{-/-}$chow vs RIP-GRP78 ${ }^{-/-}$HFD, $t$ test); (d) $S d f 2 l 1\left({ }^{*} p<0.05\right.$,

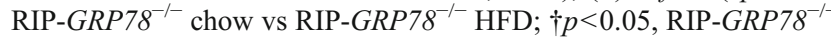
HFD vs RIP-GRP7 $78^{-1+}$ HFD, one-way ANOVA and Tukey post hoc test); (e) Chop. f Islets from $n=3$ mice per group immunoblotted for the indicated proteins (-/-, RIP-GRP $78^{-/-} ;-/+$, RIP-GRP $78^{-/+}$). Relative GRP78 (g) and phospho-EIF2 $\alpha$ (h) protein levels quantified by band

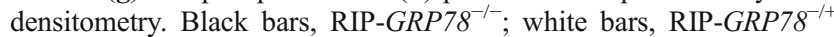
$\left({ }^{*} p<0.05\right.$, RIP-GRP $78^{-/-}$chow vs RIP-GRP7 $8^{-/-}$HFD, $t$ test). Male mice were used for the studies shown in this figure a

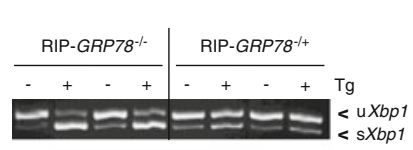

b

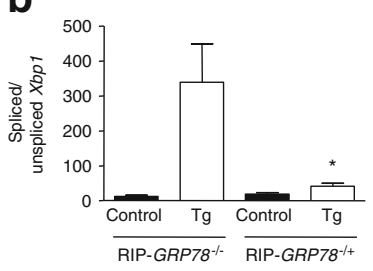

C

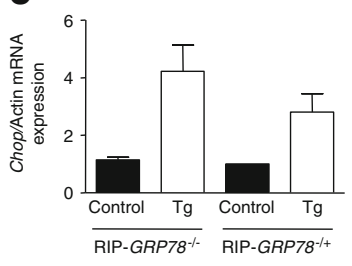

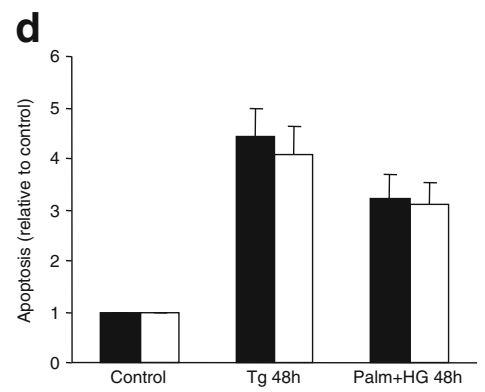

Fig. 6 Islets from RIP-GRP78 ${ }^{-/+}$mice (strain No. 3068) are resistant

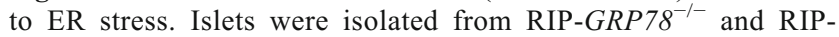
$G R P 78^{-/+}$mice and were treated or not treated with $1 \mu \mathrm{mol} / 1$ thapsigargin $(\mathrm{Tg})$ for $1 \mathrm{~h}$; levels of spliced $(\mathrm{s} X b p 1)$ and unspliced $X b p 1$ (uXbpl) were detected by RT-PCR (a), spliced/unspliced Xbpl band intensities were quantified (b) and Chop mRNA expression was analysed by real-time PCR (c). Data are from islets isolated from $n=3$ or 4 e

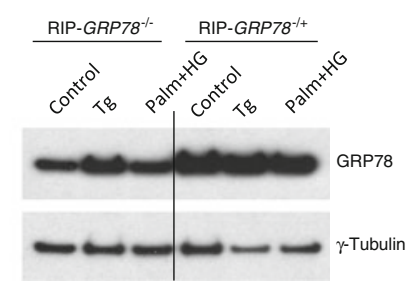

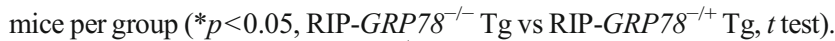
d Islets isolated from RIP- $G R P 78^{-1-}$ mice (black bars) or transgenic RIP$G R P 78^{-/+}$mice (white bars) were exposed to $\mathrm{Tg}(1 \mu \mathrm{mol} / \mathrm{l}, 48 \mathrm{~h})$ or high glucose $(\mathrm{HG}, 20 \mathrm{mmol} / \mathrm{l})+$ palmitate $(\mathrm{Palm}, 0.5 \mathrm{mmol} / \mathrm{l}$ complexed with $0.5 \%$ BSA) for $48 \mathrm{~h}$. Apoptosis was measured using a Cell Death Detection ELISA Kit (islets from $n=3$ mice per group). e Islets immunoblotted for GRP78 and $\gamma$-tubulin 
increased in response to the HFD in islets from control mice (Fig. 5d), whereas the levels of the pro-apoptotic ER-stressinducible Chop transcription factor were unchanged (Fig. 5e). As expected, Grp 78 mRNA and protein levels remained significantly elevated in transgene-positive mice in both control and HFD conditions. However, Sdf2ll expression was lower in islets from transgenic mice compared with control mice on the HFD (Fig. 5d). Levels of islet phosphorylated eukaryotic initiation factor $2 \alpha$ subunit (EIF2 $\alpha$ ) protein also tended to be reduced in RIP-GRP $78^{-1+}$ mice on the HFD (Fig. $5 \mathrm{f}$ and $\mathrm{h}$ ). Overall, these data indicate the HFD induces mild ER stress in islets and that GRP78 overproduction likely protects islets against HFD-induced ER stress.

To clearly establish that overproduction of GRP78 in transgenic islets provides protection from ER stress we examined ER stress markers in isolated islets exposed to thapsigargin. In response to thapsigargin, islets from RIP-GRP $78^{-1+}$ mice showed significantly less $X b p 1$ splicing (indicative of inositol requiring enzyme 1 [IRE1 $\alpha$ ] activation) (Fig. $6 a$ and $b$ ) and less Chop mRNA induction (indicative of double-stranded RNA-activated protein kinase [PKR]-like ER kinase [PERK] activation) (Fig. 6c), compared with islets from transgenenegative mice. Thus, the ER stress response is reduced in transgenic islets, indicating that the cells are protected from ER stress. We also examined whether isolated islets from control and transgenic mice were protected from apoptosis induced by thapsigargin or high glucose/palmitate. However, under the experimental conditions used no difference in apoptosis was observed (Fig. 6d and e).

\section{Discussion}

ER stress has been linked to obesity-induced type 2 diabetes in both insulin-sensitive tissues and in pancreatic beta cells [6]. In islets, elevated markers of ER stress have been observed in various models of rodent obesity and in humans with type 2 diabetes [9-11, 13, 14]. Thus, ER stress in beta cells has been hypothesised to contribute to beta cell dysfunction that leads to the development of type 2 diabetes $[6,33]$. In this study we present data that supports this hypothesis. Increasing ER stress resistance in pancreatic beta cells by genetically enhancing the levels of the ER chaperone GRP78 in transgenic mice resulted in significant protection against glucose intolerance, insulin resistance and hyperinsulinaemia associated with chronic high-fat feeding.

An obvious question is whether all these beneficial effects are due to enhanced resistance to ER stress afforded by GRP78 overproduction in pancreatic beta cells. Indeed, ER stress was detected in pancreatic islets in response to an HFD in control mice, which is consistent with previous observations [13]. This indicates that the UPR in islets has attempted to adjust ER homeostasis to respond to the HFD.
The adjustment is required and maintained due to ongoing cellular stress known to be associated with obesity, including increased blood NEFA levels, inflammatory stress and hyperglycaemia [2, 3]. This results in a state of insulin resistance, which was evident in the HFD mice and was accompanied by increased beta cell insulin content and hyperinsulinaemia. The UPR adjustment in pancreatic beta cells, however, is not sufficient to maintain normal glucose tolerance in the $\mathrm{C} 57 \mathrm{BL} / 6$ genetic background and male mice progressively deteriorate to the diabetic state.

In mice with enhanced GRP78 levels pancreatic beta cells are primed for responding to ER stress. This is akin to the concept of hormesis [34], whereby the extent of an adaptive response to stress determines the extent of protection against the stress. Since GRP78 levels are increased from the start (especially in strain No. 3068) the defence afforded to the pancreatic beta cells makes them more capable of counteracting the damaging effects of metabolic stress. The net effect is that the beta cells are less stressed than their wild-type counterparts in the face of ongoing metabolic stress; this results in more normal and functional beta cells and the mice have improved glucose tolerance.

How does enhancement of ER chaperone capacity and resistance to ER stress improve beta cell function? Reduced GSIS due to prolonged HFD has been associated with reduced total and cell-surface levels of the beta cell glucose transporter GLUT2, which is essential for GSIS [35, 36]. HFD-induced ER stress is likely to contribute to this defect, since GLUT2 is a transmembrane protein synthesised in the ER. Indeed, the cell-surface GLUT2 level is maintained in transgenic mice despite the HFD, which likely contributes to improved pancreatic beta cell function in GRP78 transgenic mice. However, other factors may also contribute to improved beta cell function, such as maintenance of proper proinsulin folding [20] or, indeed, maintenance of other proteins that are synthesised in the ER that have an impact on beta cell function, such as the glucagon-like peptide-1 (GLP-1) receptor [37] and/or the ATP-binding cassette transporter (ABCG1) that are essential for normal beta cell function [38].

Improved pancreatic beta cell function in transgenic mice on HFD is evident from the improved glucose tolerance and insulin response during the IPGTT. However, if GRP78 is only overproduced in beta cells, why do male mice in general gain less weight (despite no change in food intake), have reduced fat accumulation and have improved insulin tolerance, which are effects controlled by other tissues? We speculate that all these effects relate to the fact that pancreatic beta cells maintain their function despite the presence of the HFD, whereas their wild-type counterparts progressively succumb to the cellular stress and become dysfunctional. The reduced hyperinsulinaemia in transgenic mice on the HFD likely contributes to the improved insulin sensitivity. 
This hypothesis supports the model proposed by Kahn and colleagues, which links obesity, insulin resistance and type 2 diabetes to a critical role of impaired insulin secretion by pancreatic beta cells [3]. Our results provide additional support to this concept since maintaining beta cell function by enhancing ER stress resistance protects against pathological effects of obesity. This is consistent with a recent study showing that beta cell-specific overproduction of the GLUT2 transporter or GlcNAcT-IV glycosyltransferase (GNT-4A, which promotes cell-surface residency of GLUT2) improves both glucose tolerance and insulin sensitivity in mice on an HFD [35].

The effect on body-weight gain and the reduced adiposity in mice overproducing GRP78 in beta cells was also unexpected. However, this is unlikely to account for their improved glycaemic control. Mice from strain No. 3064 (approximately twofold GRP78 overproduction) also had a reduction in body weight on the HFD, but were less protected against glucose intolerance and insulin resistance. It is possible that in the RIP-GRP7 $78^{-/+}$mouse the effect on weight gain may relate to insulin's known effects in the hypothalamus (i.e. controlling body weight by reducing food intake and increasing energy expenditure) [39, 40]. We did not observe a significant effect on food intake in control vs transgenic male mice, thus perhaps increased energy expenditure or reduced hyperinsulinaemia may account for the difference in body-weight gain and fat accumulation.

In summary, we found that $\mathrm{C} 57 \mathrm{BL} / 6$ mice overproducing GRP78 in pancreatic beta cells were protected against the glucose intolerance and insulin resistance caused by obesity. By enhancing ER stress resistance pancreatic beta cell function is maintained, which ultimately improves whole-body glucose homeostasis. Thus, ER stress is a major factor in pancreatic beta cell dysfunction in obesity and approaches to reduce ER stress in pancreatic beta cells is likely to be a viable therapeutic strategy to treat type 2 diabetes or prevent its development.

Acknowledgements We thank R. Prywes (Columbia University) for the human GRP78-Myc-KDEL construct, P. Ohashi (Ontario Cancer Centre) for the RIP construct, L. Baggio (Samuel Lunenfeld Research Institute, Mt Sinai Hospital) for help with physiological measurements and islet area analysis and M. Gertsenstein (TCP) for help with transgenic mouse generation. We also thank D. Drucker (Samuel Lunenfeld Research Institute, Mt Sinai Hospital) and P. Bilan (The Hospital for Sick Children, Toronto) for comments on the manuscript.

Funding This work was funded by CIHR grants (MOP-86641 and MOP-114992) to A. Volchuk. T. Teodoro-Morrison was funded in part by an NSERC graduate studentship. A. Volchuk is a recipient of a Canada Research Chair in Diabetes Research.

Duality of interest The authors declare that there is no duality of interest associated with this manuscript.
Contribution statement TTM helped design the experiments, generated data and wrote, reviewed and edited the manuscript. IS contributed to experimental data and wrote, reviewed and edited the manuscript. LZ contributed to experimental data and reviewed and edited the manuscript. DDB contributed experimental expertise, data and revisions of the manuscript. AV contributed to the experimental plan, supervision of the project, interpretation of the data and writing the manuscript. All authors approved the final version of the manuscript.

\section{References}

1. Kahn SE, Zraika S, Utzschneider KM, Hull RL (2009) The beta cell lesion in type 2 diabetes: there has to be a primary functional abnormality. Diabetologia 52:1003-1012

2. Gregor MF, Hotamisligil GS (2011) Inflammatory mechanisms in obesity. Annu Rev Immunol 29:415-445

3. Kahn SE, Hull RL, Utzschneider KM (2006) Mechanisms linking obesity to insulin resistance and type 2 diabetes. Nature $444: 840$ 846

4. O'Rahilly S (2009) Human genetics illuminates the path to metabolic disease. Nature 462:307-314

5. Poitout V, Robertson RP (2008) Glucolipotoxicity: fuel excess and beta-cell dysfunction. Endocr Rev 29:351-366

6. Eizirik DL, Cardozo AK, Cnop M (2008) The role for endoplasmic reticulum stress in diabetes mellitus. Endocr Rev 29:42-61

7. Hotamisligil GS, Erbay E (2008) Nutrient sensing and inflammation in metabolic diseases. Nat Rev Immunol 8:923-934

8. Ron D, Walter P (2007) Signal integration in the endoplasmic reticulum unfolded protein response. Nat Rev Mol Cell Biol 8:519-529

9. Huang CJ, Haataja L, Gurlo T et al (2007) Induction of endoplasmic reticulum stress-induced beta-cell apoptosis and accumulation of polyubiquitinated proteins by human islet amyloid polypeptide. Am J Physiol Endocrinol Metab 293:E1656-E1662

10. Laybutt DR, Preston AM, Akerfeldt MC et al (2007) Endoplasmic reticulum stress contributes to beta cell apoptosis in type 2 diabetes. Diabetologia 50:752-763

11. Matveyenko AV, Gurlo T, Daval M, Butler AE, Butler PC (2009) Successful versus failed adaptation to high-fat diet-induced insulin resistance: the role of IAPP-induced beta-cell endoplasmic reticulum stress. Diabetes 58:906-916

12. Ozcan U, Cao Q, Yilmaz E et al (2004) Endopasmic reticulum stress links obesity, insulin action, and type 2 diabetes. Science 306:457-461

13. Sachdeva MM, Claiborn KC, Khoo C et al (2009) Pdx1 (MODY4) regulates pancreatic beta cell susceptibility to ER stress. Proc Natl Acad Sci U S A 106:19090-19095

14. Yusta B, Baggio LL, Estall JL et al (2006) GLP-1 receptor activation improves beta cell function and survival following induction of endoplasmic reticulum stress. Cell Metab 4:391-406

15. Ozcan U, Yilmaz E, Ozcan L et al (2006) Chemical chaperones reduce ER stress and restore glucose homeostasis in a mouse model of type 2 diabetes. Science 313:1137-1140

16. Kars M, Yang L, Gregor MF et al (2010) Tauroursodeoxycholic acid may improve liver and muscle but not adipose tissue insulin sensitivity in obese men and women. Diabetes 59:1899-1905

17. Xiao C, Giacca A, Lewis GF (2011) Sodium phenylbutyrate, a drug with known capacity to reduce endoplasmic reticulum stress, partially alleviates lipid-induced insulin resistance and beta-cell dysfunction in humans. Diabetes 60:918-924

18. Dudek J, Benedix J, Cappel S et al (2009) Functions and pathologies of $\mathrm{BiP}$ and its interaction partners. Cell Mol Life Sci 66:1556-1569 
19. Zhang L, Lai E, Teodoro T, Volchuk A (2009) GRP78, but not protein-disulfide isomerase, partially reverses hyperglycemiainduced inhibition of insulin synthesis and secretion in pancreatic $\beta$-cells. J Biol Chem 284:5289-5298

20. Scheuner D, Vander Mierde D, Song B et al (2005) Control of mRNA translation preserves endoplasmic reticulum function in beta cells and maintains glucose homeostasis. Nat Med 11:757-764

21. Walter P, Ron D (2011) The unfolded protein response: from stress pathway to homeostatic regulation. Science 334:1081-1086

22. Gorbatyuk MS, Knox T, LaVail MM et al (2010) Restoration of visual function in $\mathrm{P} 23 \mathrm{H}$ rhodopsin transgenic rats by gene delivery of BiP/Grp78. Proc Natl Acad Sci U S A 107:5961-5966

23. Kammoun LH, Chabanon H, Hainault I et al (2009) GRP78 expression inhibits insulin and ER stress-induced SREBP-1c activation and reduces hepatic steatosis in mice. J Clin Investig 119:1201-1215

24. Reddy RK, Mao C, Baumeister P, Austin RC, Kaufman RJ, Lee AS (2003) Endoplasmic reticulum chaperone protein GRP78 protects cells from apoptosis induced by topoisomerase inhibitors: role of ATP binding site in suppression of caspase-7 activation. J Biol Chem 278:20915-20924

25. Maris M, Waelkens E, Cnop M et al (2011) Oleate-induced beta cell dysfunction and apoptosis: a proteomic approach to glucolipotoxicity by an unsaturated fatty acid. J Proteome Res 10:3372-3385

26. Ohashi PS, Oehen S, Buerki K et al (1991) Ablation of "tolerance" and induction of diabetes by virus infection in viral antigen transgenic mice. Cell 65:305-317

27. Kilkenny D, Rocheleau J (2008) Fibroblast growth factor receptor1 signaling in pancreatic islet beta-cells is modulated by the extracellular matrix. Mol Endocrinol 22:196-205

28. Wijesekara N, Krishnamurthy M, Bhattachejee A, Suhail A, Sweeney G, Wheeler MB (2010) Adiponectin-induced ERK and Akt phosphorylation protects against pancreatic beta cell apoptosis and increases insulin gene expression and secretion. J Biol Chem 285:33623-33631

29. Vellanki RN, Zhang L, Guney MA, Rocheleau JV, Gannon M, Volchuk A (2010) OASIS/CREB3L1 induces expression of genes involved in extracellular matrix production but not classical endoplasmic reticulum stress response genes in pancreatic betacells. Endocrinology 151:4146-4157

30. Hartil K, Vuguin PM, Kruse M et al (2009) Maternal substrate utilization programs the development of the metabolic syndrome in male mice exposed to high fat in utero. Pediatr Res 66:368-373

31. Fukuda S, Sumii M, Masuda Y et al (2001) Murine and human SDF2L1 is an endoplasmic reticulum stress-inducible gene and encodes a new member of the Pmt/rt protein family. Biochem Biophys Res Commun 280:407-414

32. Hartley T, Siva M, Lai E, Teodoro T, Zhang L, Volchuk A (2010) Endoplasmic reticulum stress response in an INS-1 pancreatic beta-cell line with inducible expression of a folding-deficient proinsulin. BMC Cell Biol 11:59

33. Kaufman RJ, Back SH, Song B, Han J, Hassler J (2010) The unfolded protein response is required to maintain the integrity of the endoplasmic reticulum, prevent oxidative stress and preserve differentiation in beta-cells. Diab Obes Metab 12(suppl 2):99-107

34. Kolb H, Eizirik DL (2011) Resistance to type 2 diabetes mellitus: a matter of hormesis? Nat Rev Endocrinol 8:183-192

35. Ohtsubo K, Chen MZ, Olefsky JM, Marth JD (2011) Pathway to diabetes through attenuation of pancreatic beta cell glycosylation and glucose transport. Nat Med 17:1067-1075

36. Ohtsubo K, Takamatsu S, Minowa MT, Yoshida A, Takeuchi M, Marth JD (2005) Dietary and genetic control of glucose transporter 2 glycosylation promotes insulin secretion in suppressing diabetes. Cell 123:1307-1321

37. Baggio LL, Drucker DJ (2007) Biology of incretins: GLP-1 and GIP. Gastroenterology 132:2131-2157

38. Sturek JM, Castle JD, Trace AP et al (2010) An intracellular role for ABCG1-mediated cholesterol transport in the regulated secretory pathway of mouse pancreatic beta cells. J Clin Invest 120:2575-2589

39. Sisley S, Sandoval D (2011) Hypothalamic control of energy and glucose metabolism. Rev Endocr Metab Discord 12:219-233

40. Schwartz MW, Woods SC, Porte DJ, Seeley RJ, Baskin DG (2000) Central nervous system control of food intake. Nature 404:661671 